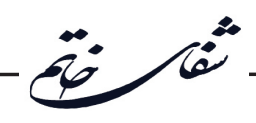

\title{
Early Maladaptive Schemas in Patients with Psychosomatic Disorder and Multiple Sclerosis
}

\section{Davood Manavipour*, Laya Sadat Miri}

Department of Psychology, Faculty of Psychology and Educational Sciences, Garmsar Branch, Islamic Azad University, Garmsar, Iran

\section{ABSTRACT}

Introduction: According to the importance of psychological factors in psychosomatic disorders and multiple sclerosis (MS) as well as its importance in early maladaptive schemas in psychological disorders, the present study was aimed to evaluate early maladaptive schemas in patients with psychosomatic disorders and MS and compared that with healthy subjects. Materials and Methods: Research method was ex post facto. This study evaluated 100 patients (50 with MS and 50 with psychosomatic disorder) and compared them to 50 healthy people that completed Young Schema Questionnaire (short form). The study was causalcomparative. Results: The results showed that the average score of vulnerability to harm or illness subscale in people with psychosomatic disorder was higher than healthy people and the average score of emotional deprivation, dependence/incompetence, and failure subscale in patients with MS was higher than healthy group. Conclusion: Early maladaptive schemas should be considered as an important element when comparing patients with psychosomatic disorders and MS with healthy people.

\section{Key words:}

1. Psychosomatic Medicine

2. Multiple Sclerosis

3. Psychophysiologic Disorders

\section{*Corresponding Author: Davood Manavipour}

E-mail: manavipor53@yahoo.com

doi: 10.18869/acadpub.shefa.5.1.40 


\section{طر حوارههاى ناساز كار اوليه در بيماران با اختلال روانتنى و مالتيبِل اسكلروز}

$$
\text { داود معنوى يورّ", لعيا سادات ميرى }
$$

كروه روانشناسى، دانشكده روانشناسى و علوم تربيتى، واحد كرمسار، دانشعاه آزاد اسلامى، كرمسار، ايران

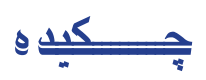

مقدمه: با توجه به اهميت عوامل روانشناختى در اختلالات روانتنى و مالتييل اسكلروز و همجنين

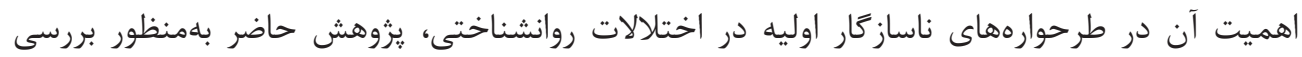

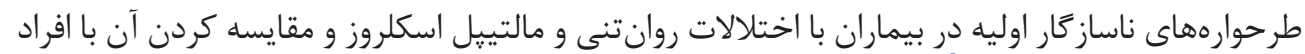

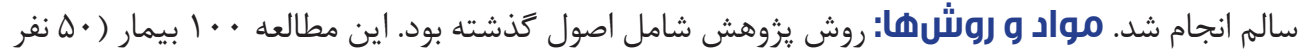

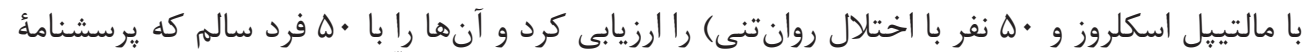

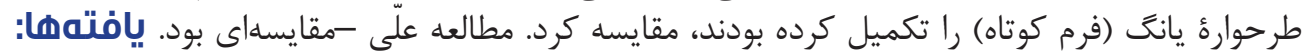

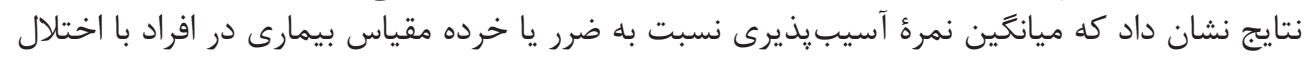

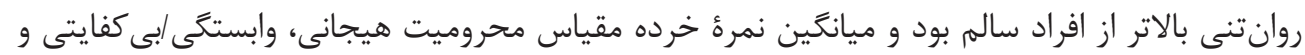

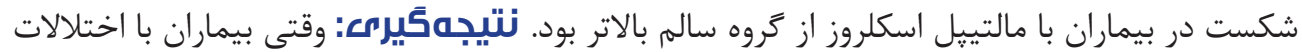

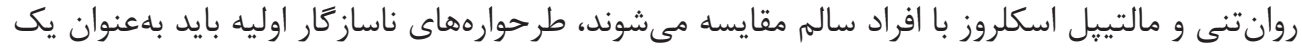
اصل مهم در نظر كرفته شوند.

\footnotetext{
كليد وازهها:

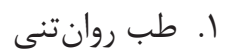
r. ب. مالتييل اسكلروز

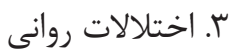
- فيزيولوزيكى اختلات
} 


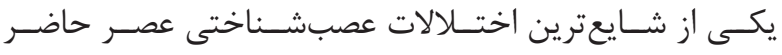

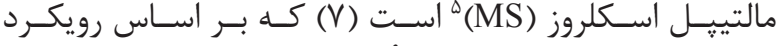

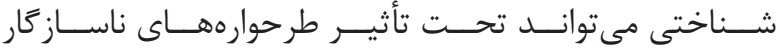

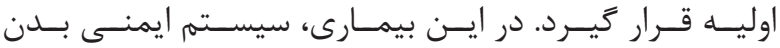

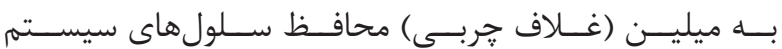

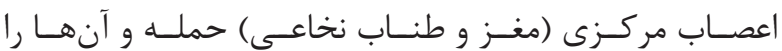

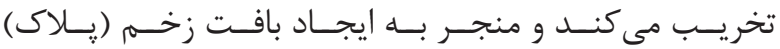

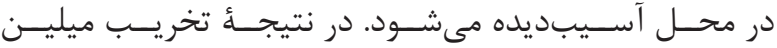

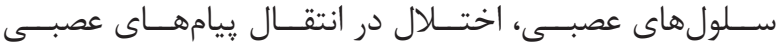

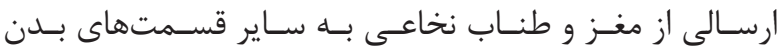

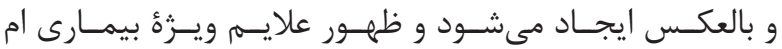

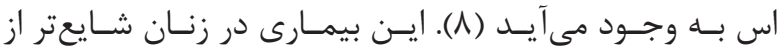

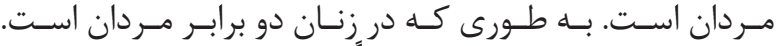

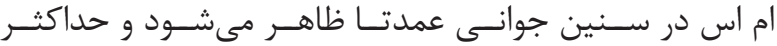

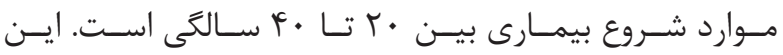

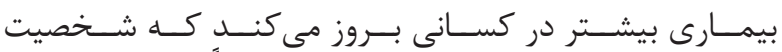

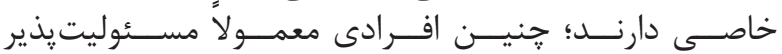

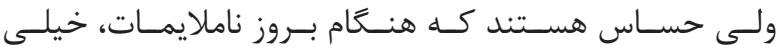

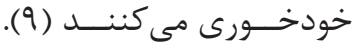

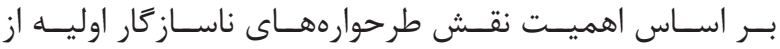

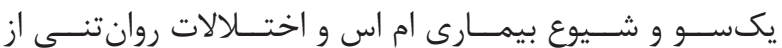

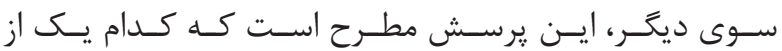

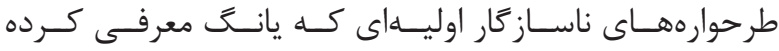

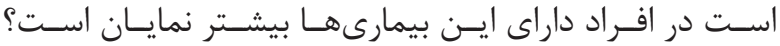

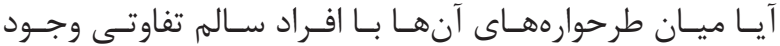

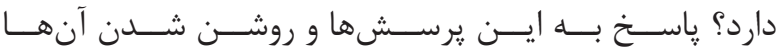

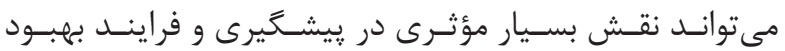

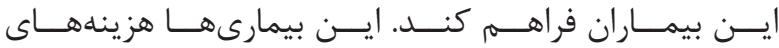

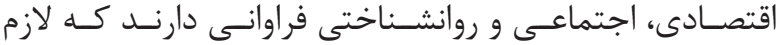

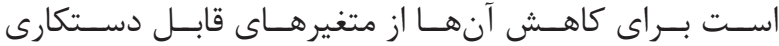

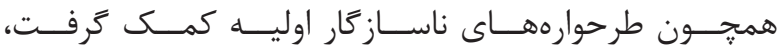

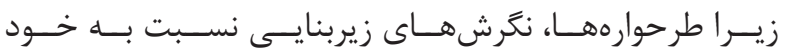

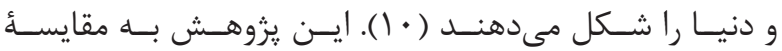

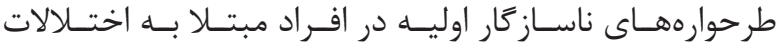

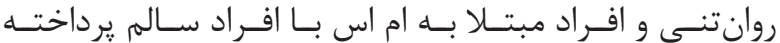

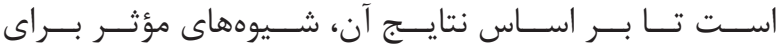

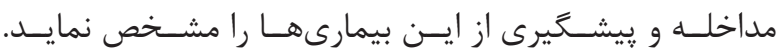

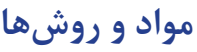

جامعلُ آمارى، آزمودنى ها و حجم نمونه

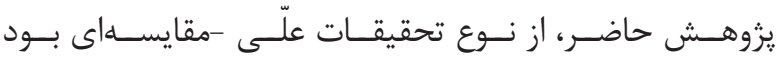

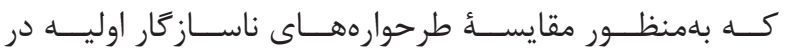

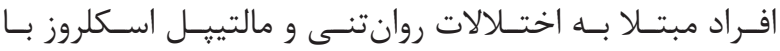

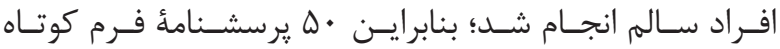

Psychosomatic

${ }^{2}$ Diagnostic and statistical manual of mental disorders

${ }^{3}$ Early Maladaptive Schemas

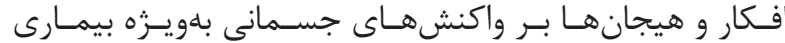

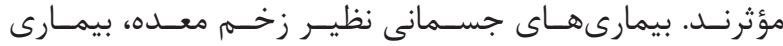

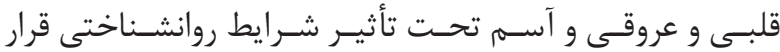

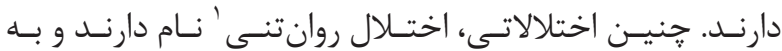

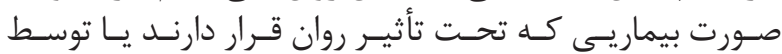

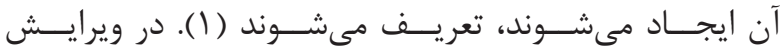

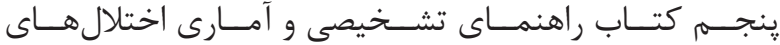

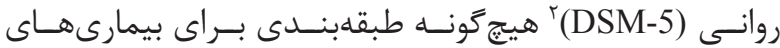

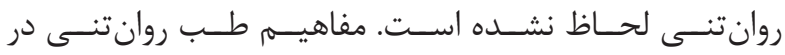

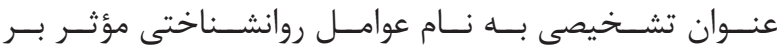

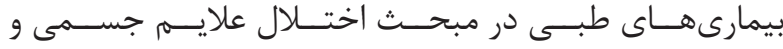

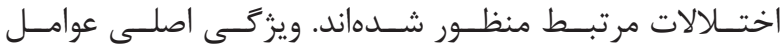

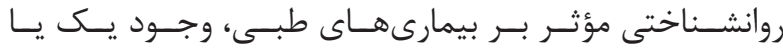

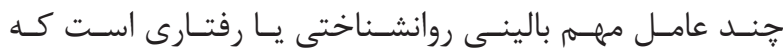

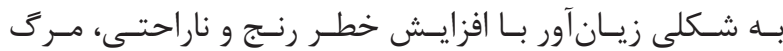

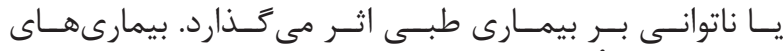

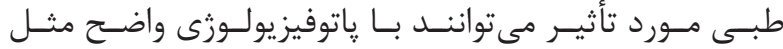

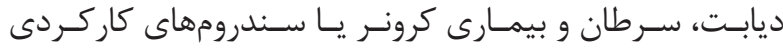

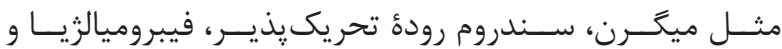

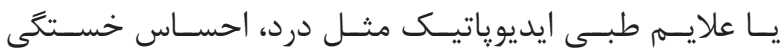

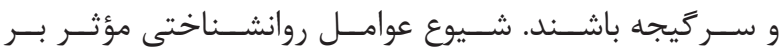

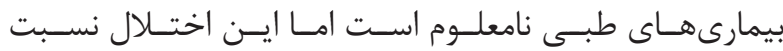

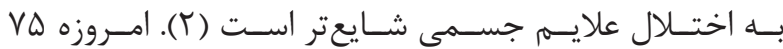

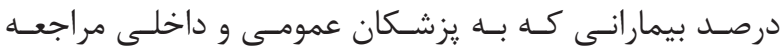

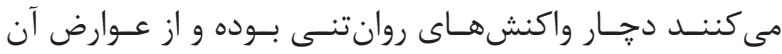

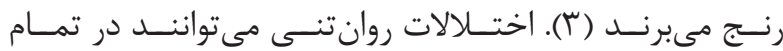

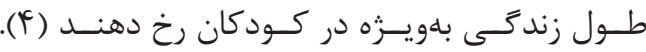

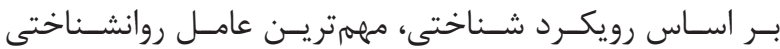

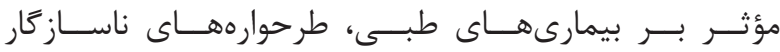

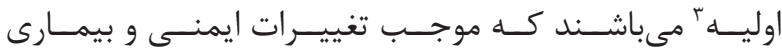

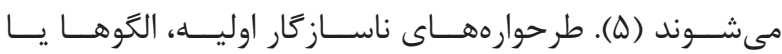

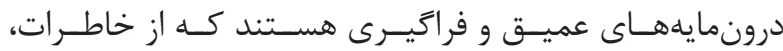

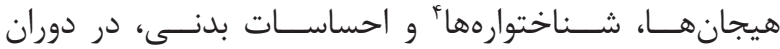

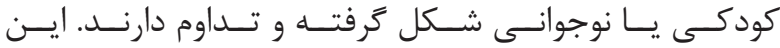

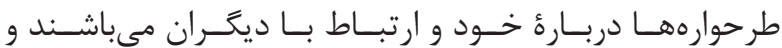

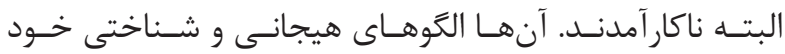

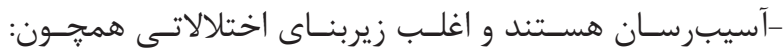

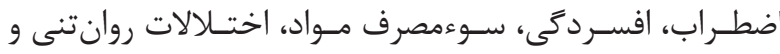

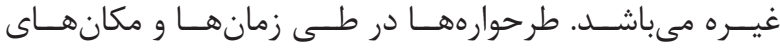

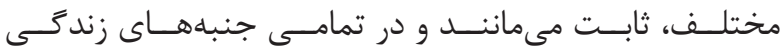

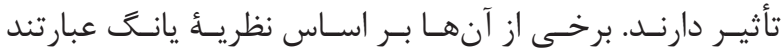

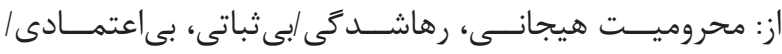

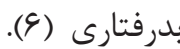

${ }^{4}$ Cognitions

${ }^{5}$ Multiple sclerosis 


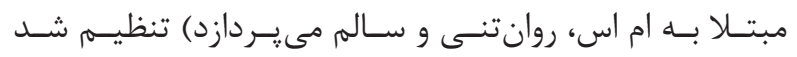

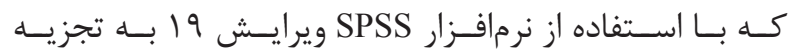

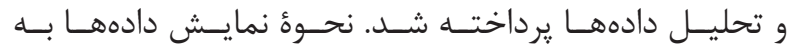

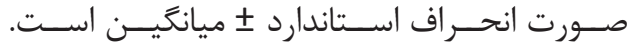

بافته ها

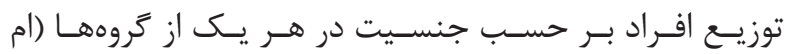

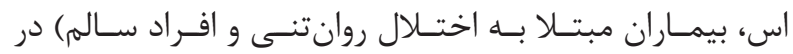

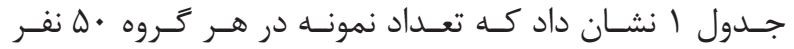

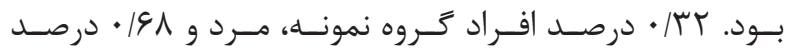

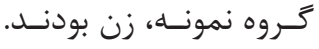

جدول ا- توزيع فراوانى نمونه برحسب جنسيت.

\begin{tabular}{|c|c|c|}
\hline درصد فراوانى & فراوانى & جنسيث \\
\hline . & 19 & مرد \\
\hline $.19 \Lambda$ & r & زن \\
\hline .11. & $\Delta$. & كل \\
\hline
\end{tabular}

بهمنظــور بررســى تفــاوت ميــان طرحوارههــــاى ناســاز كار

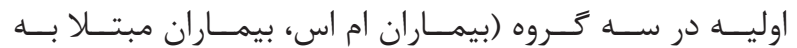

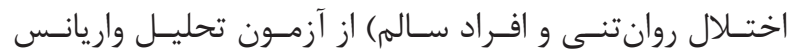

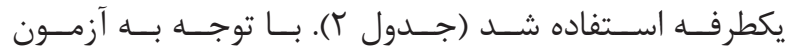

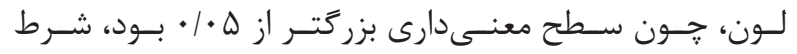

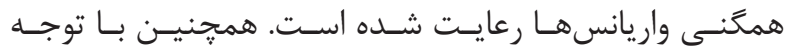

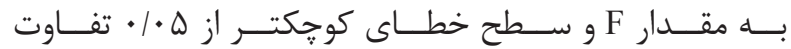

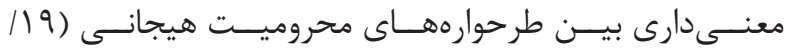

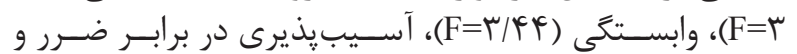

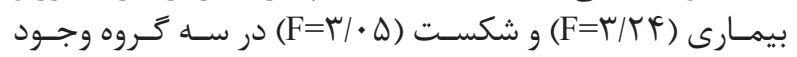

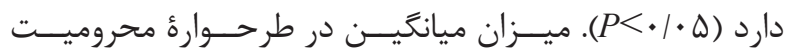

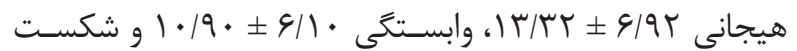

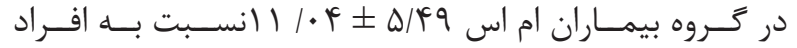

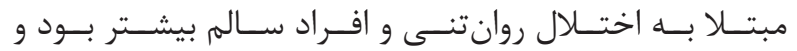

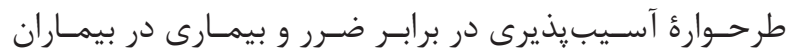

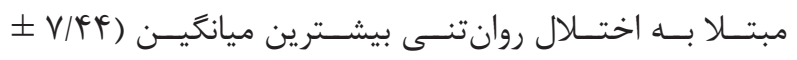

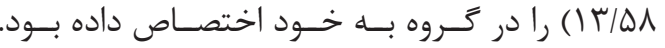

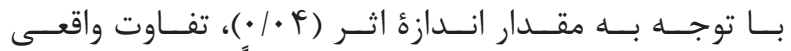

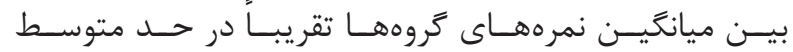

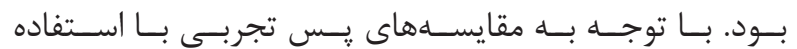

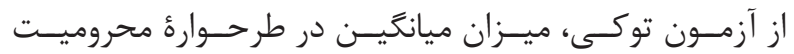

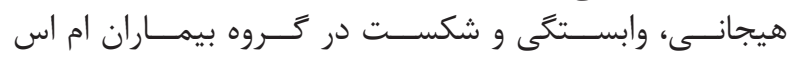

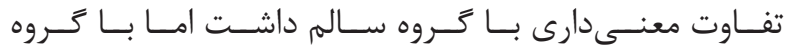

${ }^{6}$ Schema questionnaire-short form

${ }^{7}$ Schmidt NB

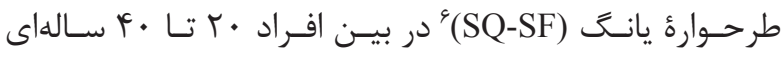

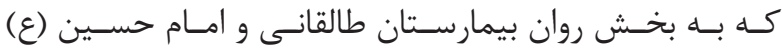

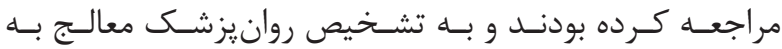

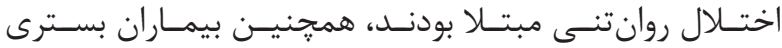

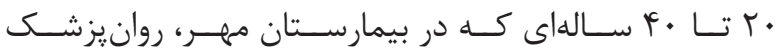

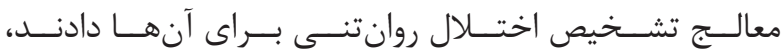

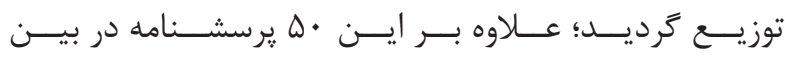

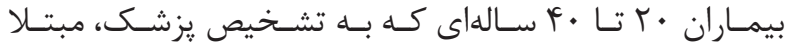

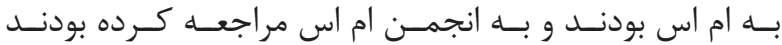

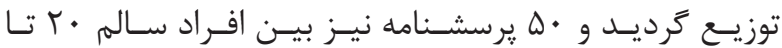

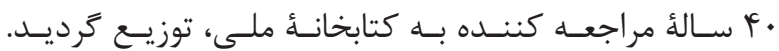

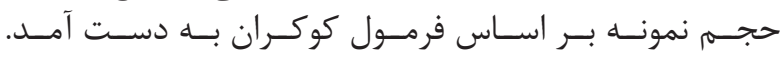

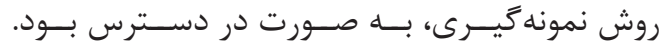

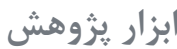

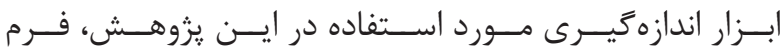

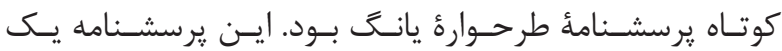

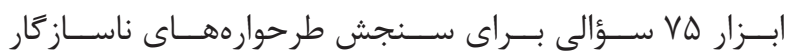

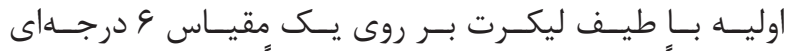

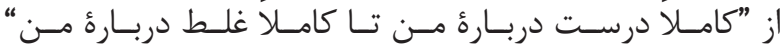

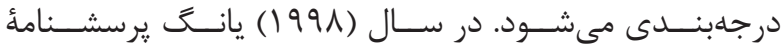

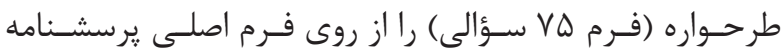

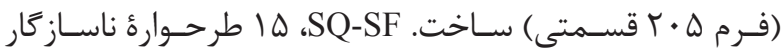

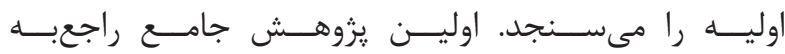

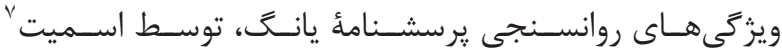

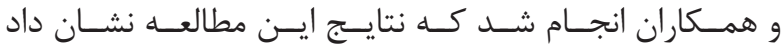

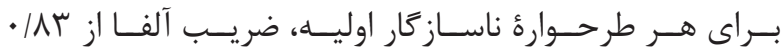

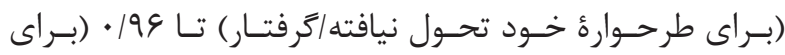

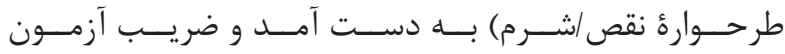

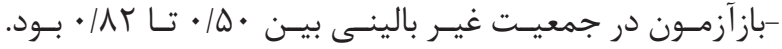

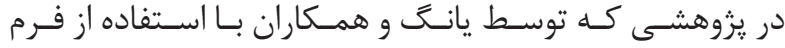

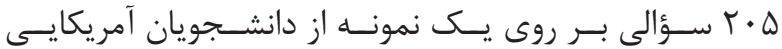

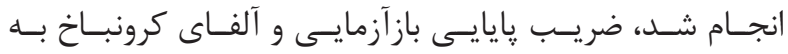

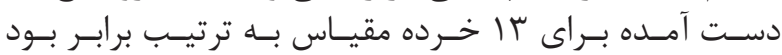

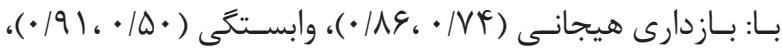

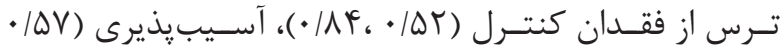

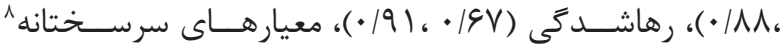

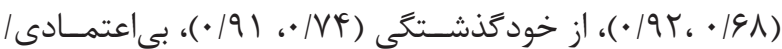

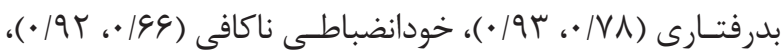

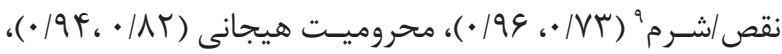

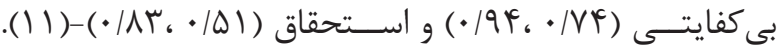

تجزيه و تحليل دادهها

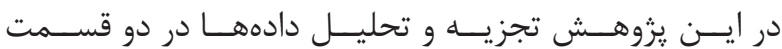

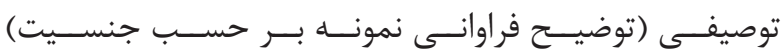

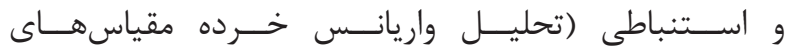

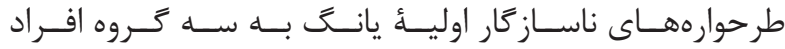

${ }^{8}$ Unrelenting standards/hypercriticalness

${ }^{9}$ Defectiveness/shame 
جدول r- تحليل واريانس خرده مقياسهاى طرحوارههاى ناساز كار اوليه در بين سه كروه (ام اس، روانتنى و سالم).

\begin{tabular}{|c|c|c|c|c|c|}
\hline sulengen & F & of & تEth & $\log x^{x}$ & 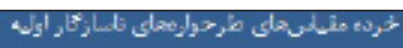 \\
\hline \multirow{3}{*}{ 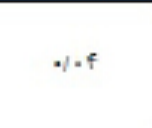 } & \multirow{3}{*}{$4 / 19$} & $914 \pi$ & ITIST & MS & \multirow{3}{*}{ 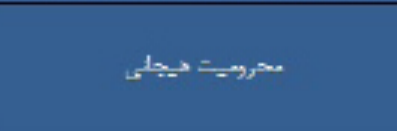 } \\
\hline & & $x \cdot 9$ & $1 \mathrm{NTT}$ & دونتش & \\
\hline & & $8 \cdot 4$ & 1.114 & سالم & \\
\hline \multirow{3}{*}{$-/ Y}$. & \multirow{3}{*}{$-\operatorname{traF}$} & $\operatorname{lin}$ & If/AY & 2.IS & \multirow{3}{*}{ رجالمدتى لي لعاتي } \\
\hline & & 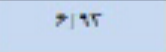 & $1 \pi / 4$ & بولثتى & \\
\hline & & $\Delta r y$ & ITIAY & ath & \\
\hline \multirow{3}{*}{.$/ 49$} & \multirow{3}{*}{$\cdot / \mathrm{Yr}$} & ator & $1 \pi 94$ & 2.15 & \multirow{3}{*}{ بي العتمادى لكدرتائى } \\
\hline & & $\$ \$ 1$ & $\pi / 1 F$ & يونتش & \\
\hline & & fivo & $1 \times 1$. & سالم & \\
\hline \multirow{3}{*}{$-1 * 4$} & \multirow{3}{*}{$-/ \mathrm{Y}$} & ows & จ*. & Mrs & \multirow{3}{*}{ 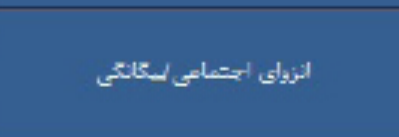 } \\
\hline & & 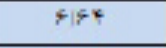 & 1.11 & رئتص & \\
\hline & & Ris & NAS & 吾 & \\
\hline \multirow{3}{*}{ ז } & \multirow{3}{*}{$\pi /-T$} & $r f \mathrm{fo}^{2}$ & N9F & MS & \multirow{3}{*}{ نقسي آشرم } \\
\hline & & 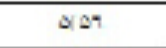 & NOA & برانتص & \\
\hline & & MFY & vitf & مالم & \\
\hline \multirow{3}{*}{.$/+1$} & \multirow{3}{*}{$\cdot / \Delta, V$} & $4 \mid T 1$ & 18106 & MS & \multirow{3}{*}{ |- } \\
\hline & & 918 & $\ln \cdot x$ & دولنتى & \\
\hline & & $8 \pi$ & 120 & مالم & \\
\hline \multirow{3}{*}{$1+4$} & \multirow{3}{*}{$r \times 4$} & $\Rightarrow 1 \mathrm{x}$ & 1.19. & MIS & \multirow{3}{*}{ 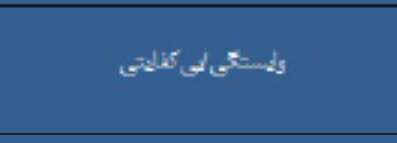 } \\
\hline & & FIDY & จ... & دانتي & \\
\hline & & Fi- & NTA & مالم & \\
\hline \multirow{3}{*}{$.1 \pi \%$} & \multirow{3}{*}{ NTT } & का9 & intक्ष & Mrs & \\
\hline & & $\rho_{/ 1 / \Delta}$ & 3154 & يدانتص & 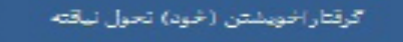 \\
\hline & & antr & $7.18 \alpha$ & alt & \\
\hline & & QA. & $191 \times-$ & M.rs & \\
\hline$\cdot[\mathrm{ry}$ & vys & $9 / \pi \cdot$ & $2+174$ & ب & ليلاعت \\
\hline & & $x_{(x)}$ & $m \cdots$ & all & \\
\hline & & 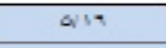 & $19 / 34$ & MIS & \\
\hline$\cdot F F$ & $-1 * 3$ & Fift & $r+\%$ & ردانتت & 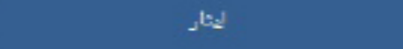 \\
\hline & & axty & $181-5$ & NL & \\
\hline & & $f / x f$ & $363^{\circ}$ & 2.15 & \\
\hline$+\%$ & TIF: & $\frac{1}{4 a}$ & $\pi+4-$ & ردانتس & 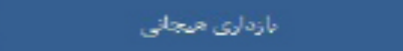 \\
\hline & & QITP & $3.18 \times$ & rle & \\
\hline & & $F / T$ & $w+4$ & M.rs & \\
\hline 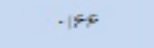 & $-1+1$ & $\bar{Y} T F$ & $14 / 5-$ & داءنتش & 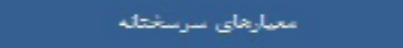 \\
\hline & & 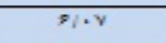 & i.298 & atl & \\
\hline & & ats & $\pi+44$ & MS & \\
\hline$\cdot \infty$ & ras & $s \rightarrow 0$ & $197=$ & 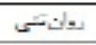 & 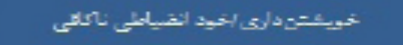 \\
\hline & & ort & $49 \times-$ & سالم & \\
\hline & & $a>v$ & ITISA & MS & \\
\hline+4 & tre & Yikt & 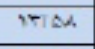 & سدانت & 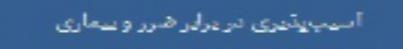 \\
\hline & & alot & $.14-$ & NL & \\
\hline & & Q149 & $3+4$ & 2.15 & \\
\hline $.1 \cdot 0$ & $T /-s$ & $6 / T$ & .142 & ردانتس & شكست \\
\hline$:$ & & $F_{i} F_{T}$ & $N+A$ & all & \\
\hline
\end{tabular}

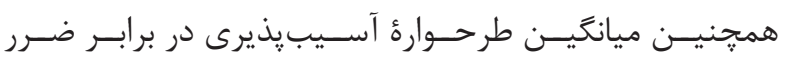

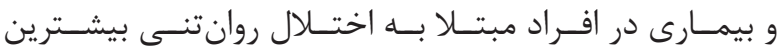

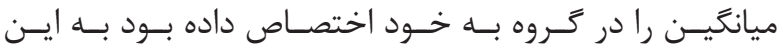

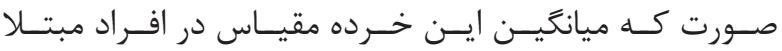

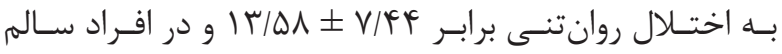

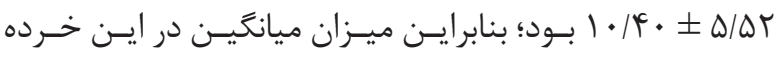

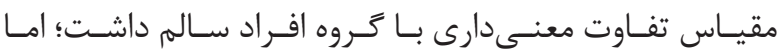

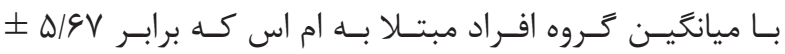

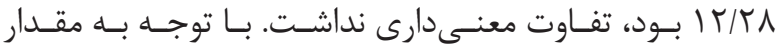

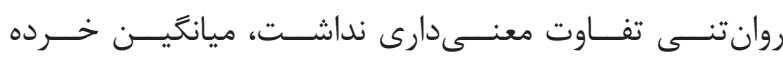

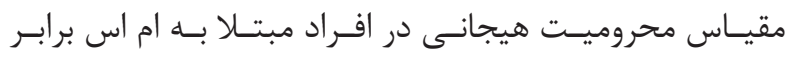

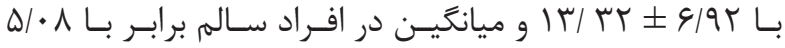

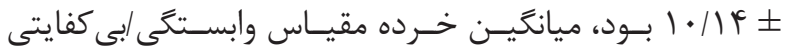

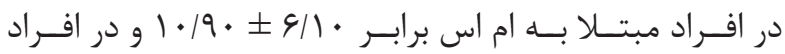

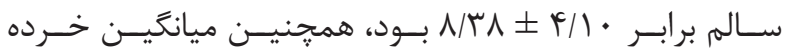

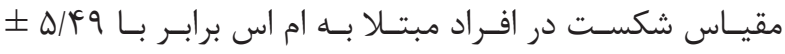

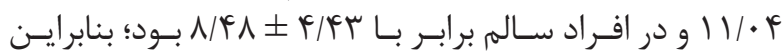

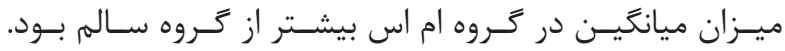




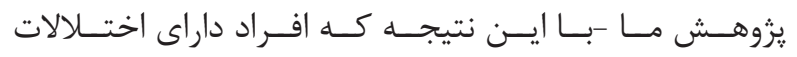

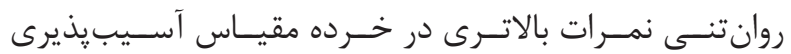

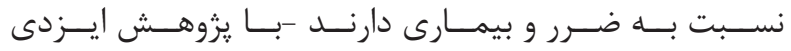

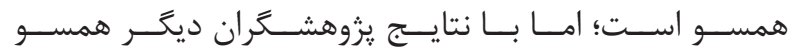

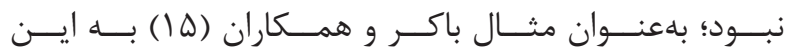

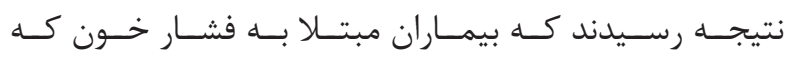

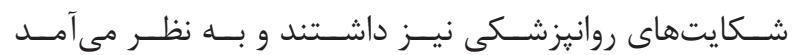

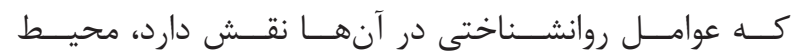

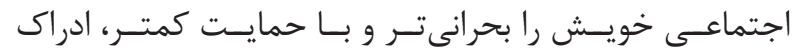

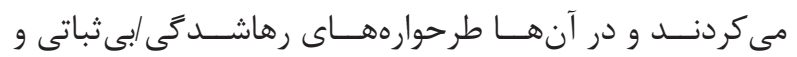

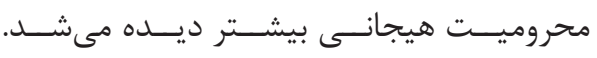

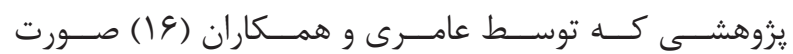

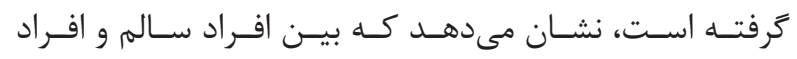

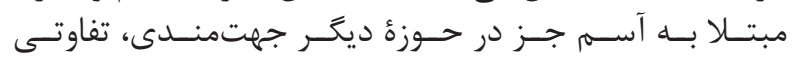

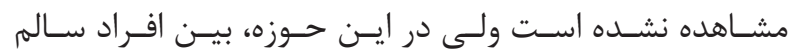

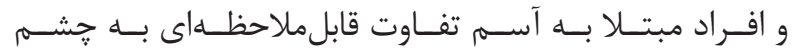

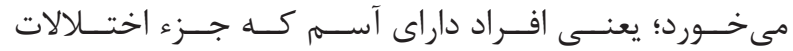

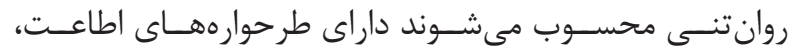

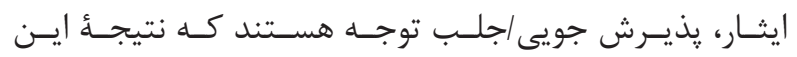

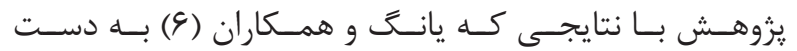

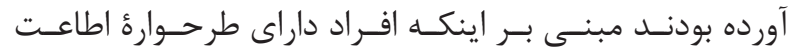

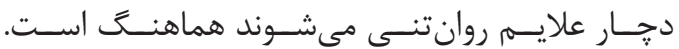

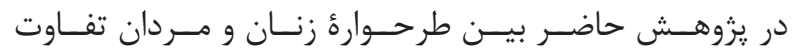

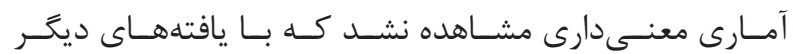

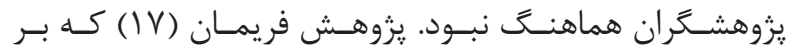

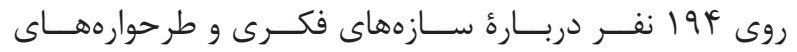

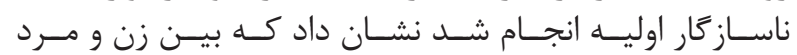

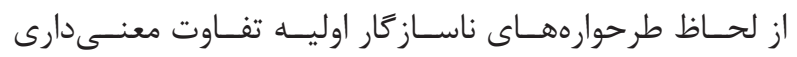

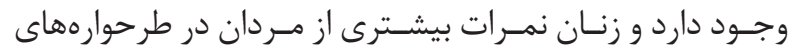

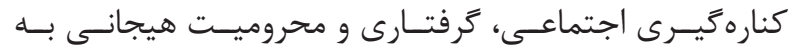

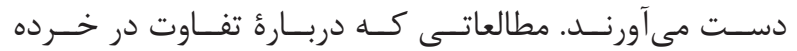

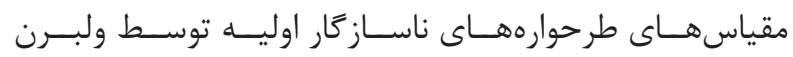

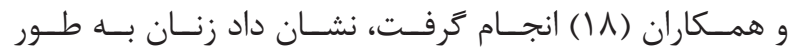

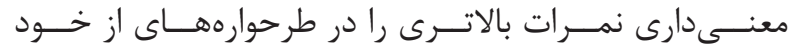

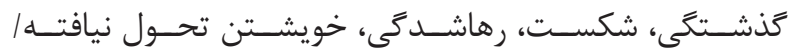

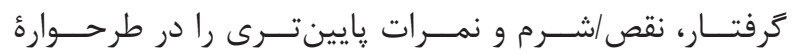

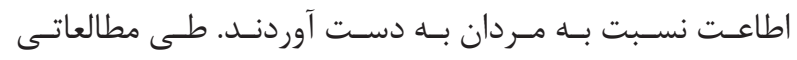

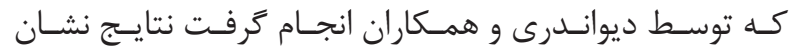

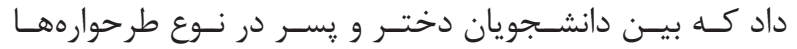

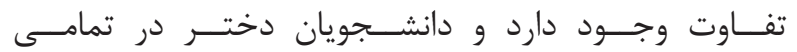

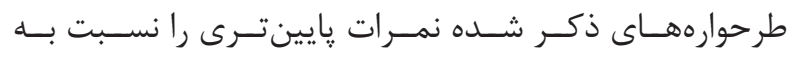

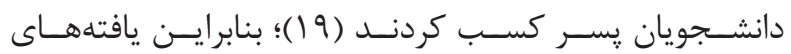

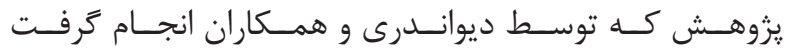

${ }^{10}$ Emotional deprivation

${ }^{11}$ Disconnection and rejection

${ }^{12}$ Dependence/incompetence

${ }^{13}$ Failure

${ }^{14}$ Impaired autonomy and performance

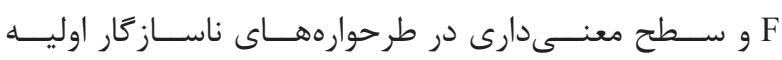

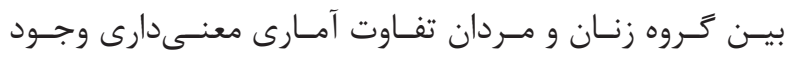
نداشـت

\section{بحث و نتيجهَ}

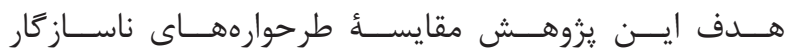

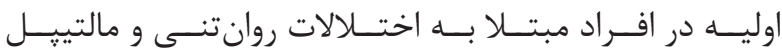

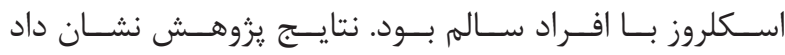

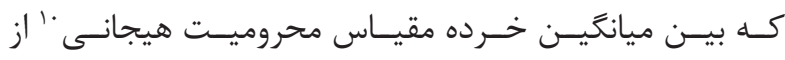

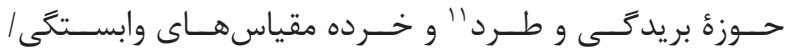

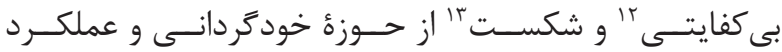

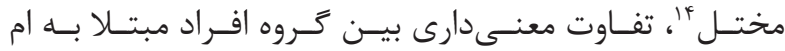

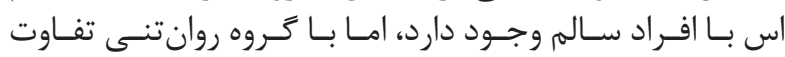

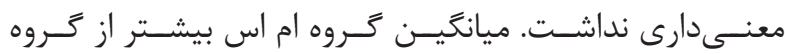

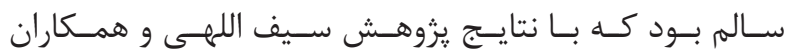

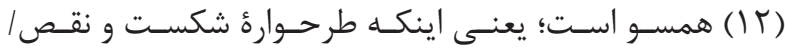

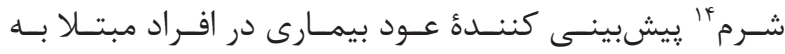

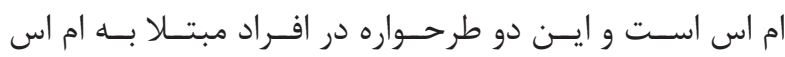

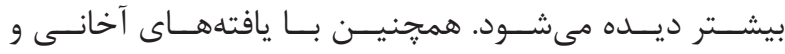

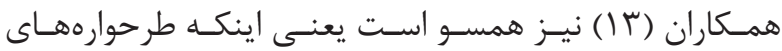

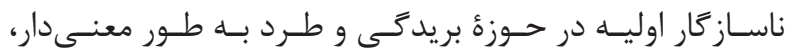

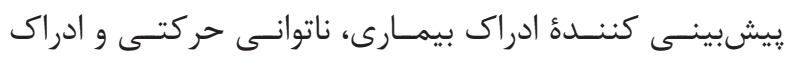

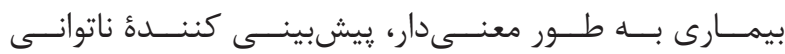

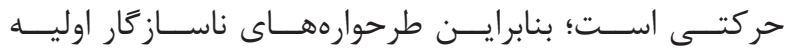

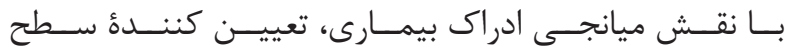

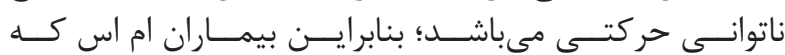

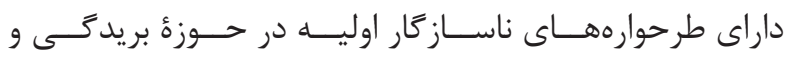

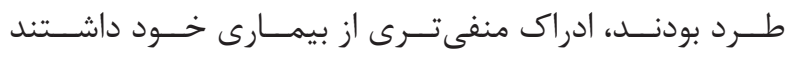

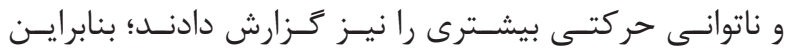

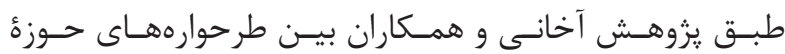

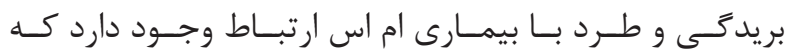

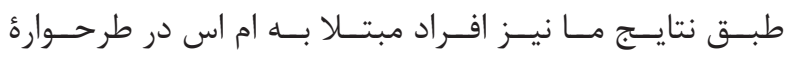

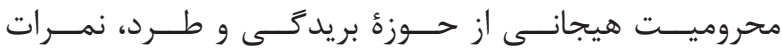

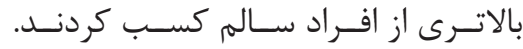

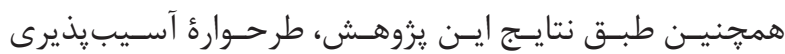

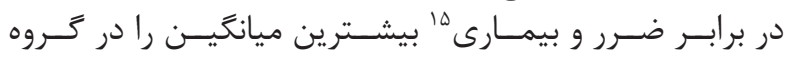

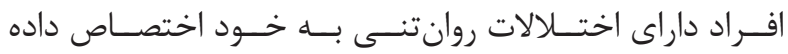

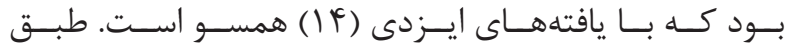

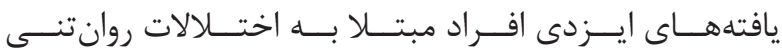

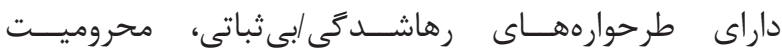

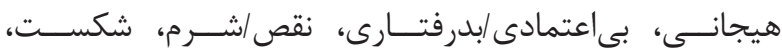

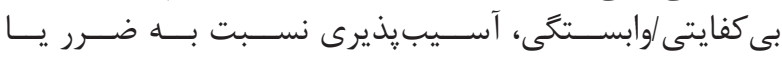

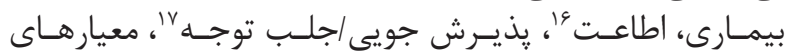

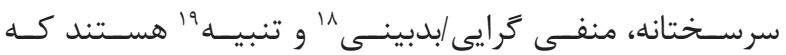

${ }^{15}$ Vulnerability to harm or illness

${ }^{16}$ Subjugation

${ }^{17}$ Approval-seeking/recognition-seeking

${ }^{18}$ Negativity/pessimism

${ }^{19}$ Punitiveness 


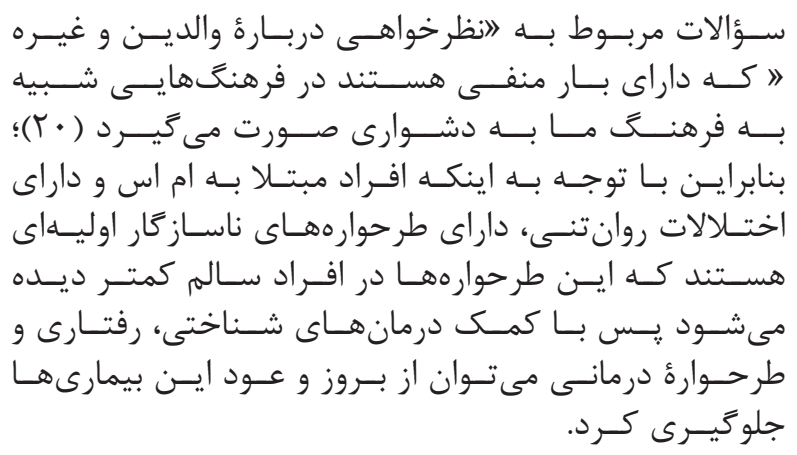

1. Seligman MEP, Walker EF, Rosenhan DL. Abnormal psychology. New York: W. W. Norton \& Company. 2001.

2. Association AP. Diagnostic and statistical manual of mental disorders (DSM). $5^{\text {th }}$ ed. American Psychiatric Association. 2013.

3. Alipur A. Investigate the relationship between personality characteristics, social support, psychosomatic vulnerability to stress: Tarbiat Modares University. 1992; 3(10): 117-62.

4. Hawton K, Salkovskis PM, Salkovskis J, Kirk J. Clark DM. Cognitive behaviour therapy for psychiatric problems, a practical guide. Oxford: Oxford University Press. 1989.

5. Gholami M, Pasha G, Sodani M. To investigate the effectiveness of group logotherapy on the increasement of life expectancy and health on female teenager major thalassemia patients of Ahvaz city. Knowledge \& Research in Applied Psychology. 2010; 11(42): 23-42.

6. Young JE, Klosko JS, Weishaar ME. Schema therapy: a practitioner's guide. New York: Guilford. 2003.

7. Farahani MN, Ahlami M. The comparison of the personality factors and locus of control in multiple sclerosis (MS) patients and normal people. J Birjand Univ Med Sci. 2005; 12(1-2): 41-7.

8. Compston A, Coles A. Multiple sclerosis. Lancet. 2008; 372(9648): 1502-17.

9. Sadock BJ, Sadock VA. Kaplan and sadock's pocket handbook of clinical psychiatry. 5th ed. Lippincott Williams and Wilkins. 2010.

10. Leahy RL. Cognitive therapy techniques: a practitioner's guide. New York: Guilford. 2008.

11. Schmidt NB, Joiner TE, Young JE, Telch MJ. The

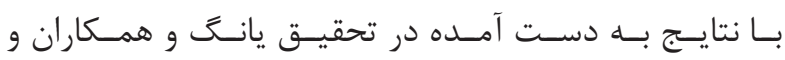

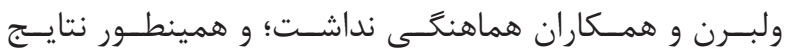

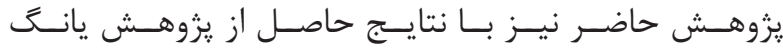

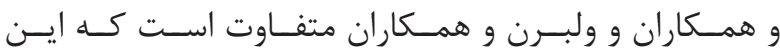

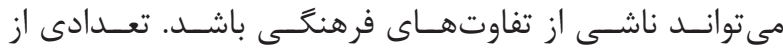

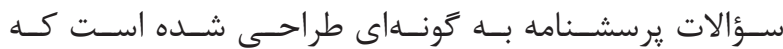

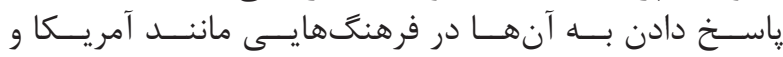

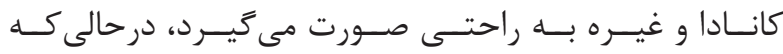

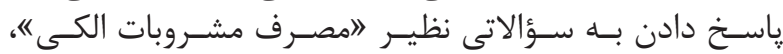

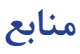

schema questionnaire: investigation of psychometric properties and the hierarchical structure of a measure of maladaptive schemas. Cognitive Therapy and Research. 1995; 19: 295-321.

12. Seifollahi ASL B, Alibeigi N, Bakhtiari E. Giardia protozoa a comparative study of early maladaptive schemas and irrational beliefs in recurrence (relapse) and psychological symptoms in male versus female with multiple sclerosis (MS). IJAPBS. 2015; 4(8): 52-63.

13. Akhani A, Izadikhah Z, Bagherian-Sararoudi R, Khorvash $\mathrm{F}$. Investigate the effect of illness perception on the relationship between early maladaptive schemas and level of motor diability in multiple sclerosis patients. J Res Behav Sci. 2013; 10(7): 609-18.

14. Izadi F. Compare early maladaptive schemas between people with psychosomatic disorder and healthy volunteer 30 to 50 years in Tehran. Abstract book of fourth international congress of psychosomatic; Isfahan: Azad University of Khorasgan. 2012.

15. Baker B, Kazarian S, Morgues J. Perceived interpersonal attitudes and psychiatric complaints in patiants with essential hypertension. J Clin Psychol. 1994; 50(3): 320-4.

16. Ameri F, Bayat B, Khosravi Z. Comparison of early maladaptive schemas and defense styles in asthmatic,alexithymic and normal subjects. IJPCP. 2014; 2(1): 88-93.

17. Freeman N. Constructive thinking and early maladaptive schemas as predictors of interpersonal adjustment and marital satisfaction. Rosemead school: Biola university. 1998.

18. Welburn K, Coristine M, Dagg P, Pontrefact A, Jordan S. The schema questionnaire-short form: factor analysis and relationship between schemas and 
symptoms. Cognit Ther Res. 2002; 26(4): 519-30.

19. Divandari H, Ahi G, Akbari H, Mahdian H. Young schema questionnaire-short form (SQ-SF): evaluate the psychometric properties and factor structure of the academic students of Islamic Azad University of Kashmar branch. Pazhouhesh-Nameye Tarbiati. 2009;
5(20): 103-33.

20. Akbari E, Bakhshipour Roudsari A, Azimi Z, Fahimi S, Ghasempour A, Amiri A. Comparison of anxiety, depression, brain behavioral systems (BIS-BAS), coping styles, anger and hostility in people with and without asthma. J Psychosom Dis. 2011; 9(5): 313-30. 